\title{
Gait in Pregnancy-related Pelvic girdle Pain: amplitudes, timing, and coordination of horizontal trunk rotations
}

\author{
Wen Hua Wu - Onno G. Meijer · Sjoerd M. Bruijn • \\ Hai Hu · Jaap H. van Dieën · Claudine J. C. Lamoth · \\ Barend J. van Royen · Peter J. Beek
}

Received: 27 November 2007 / Revised: 29 April 2008 / Accepted: 9 June 2008 / Published online: 26 July 2008

(C) The Author(s) 2008

\begin{abstract}
Walking is impaired in Pregnancy-related Pelvic girdle Pain (PPP). Walking velocity is reduced, and in postpartum PPP relative phase between horizontal pelvis and thorax rotations was found to be lower at higher velocities, and rotational amplitudes tended to be larger. While attempting to confirm these findings for PPP during pregnancy, we wanted to identify underlying mechanisms. We compared gait kinematics of 12 healthy pregnant women and 12 pregnant women with PPP, focusing on the amplitudes of transverse segmental rotations, the timing and relative phase of these rotations, and the amplitude of spinal rotations. In PPP during pregnancy walking velocity was lower than in controls, and negatively correlated with fear of movement. While patients' rotational amplitudes were larger, with large inter-individual differences, spinal
\end{abstract}

W. H. Wu - O. G. Meijer

Second Affiliated Hospital of Fujian Medical University,

Quanzhou, People's Republic of China

W. H. Wu · O. G. Meijer

Orthopaedic Biomechanics Laboratory,

Fujian Medical University, Fuzhou, Fujian,

People's Republic of China

O. G. Meijer $(\square) \cdot$ S. M. Bruijn $\cdot$ H. Hu .

J. H. van Dieën - C. J. C. Lamoth · P. J. Beek

Research Institute MOVE, Faculty of Human Movement

Sciences, Vrije Universiteit, Van der Boechorststraat 9,

1081 BT Amsterdam, The Netherlands

e-mail: o_g_meijer@fbw.vu.nl

H. $\mathrm{Hu}$

Zhongshan Hospital, University of Xiamen, Xiamen, Fujian, People's Republic of China

B. J. van Royen

Department of Orthopaedic Surgery, Research Institute MOVE,

Vrije Universiteit Medical Centre, Amsterdam, The Netherlands rotations did not differ between groups. In the patients, peak thorax rotation occurred earlier in the stride cycle at higher velocities, and relative phase was lower. The earlier results on postpartum PPP were confirmed for PPP during pregnancy. Spinal rotations remained unaffected, while at higher velocities the peak of thorax rotations occurred earlier in the stride cycle. The latter change may serve to avoid excessive spine rotations caused by the larger segmental rotations.

Keywords Pregnancy-related Pelvic girdle Pain . Gait kinematics - Transverse rotation - Trunk coordination . Relative phase

\section{Introduction}

Evidence is growing that Pregnancy-related Pelvic girdle Pain (PPP) is a distinct clinical entity, the exact causes of which still remain unknown [26, 27]. Total prevalence of PPP during pregnancy has been estimated at 22.5\% [41], with $10 \%$ of patients having mild symptoms only, $10 \%$ deserving at least some medical attention, and $2.5 \%$ having serious pain and/or disability [28].

Women with PPP suffer from deep gluteal pain [24], pain during provocation tests [9, 32], and pain in a variety of locations that often change over time in the individual patient $[10,20]$. There is strong evidence that strenuous work, previous low back pain, and previous PPP are risk factors for the emergence of PPP [41], which appears to suggest a causal role for mechanical trauma (or microtraumata), quite possibly related to the loosening of the connective tissues by the pregnancy hormone relaxin [11]. Indeed, several structural abnormalities have been observed, e.g., vertical displacement of a pubic bone 
("symphyseal step") was reported while patients were standing on one leg [22], and laxity of the sacroiliac joints appeared to be asymmetric [5], probably related to painful tension in the long dorsal ligaments [38]. Still, in different patients, and in the same patient at different times, different structures appear to be involved $[9,20]$, and the aetiology of PPP is probably multifactorial [25-27].

Women with PPP often have difficulties in performing Activities of Daily Living, such as housework, exercise, activities with the children, employment, leisure/hobbies, and personal relationships or married life [17]. Many patients cannot walk quickly or cover long distances $[6,8$, 21]. Moreover, during walking, women with PPP reported a sensation as if hip flexion were temporarily blocked (a "catching" sensation [35]). The feeling "as if the leg is paralysed" during the Active Straight Leg Raising test [22] may be related to this phenomenon. These unusual findings appear to suggest problems in the control and/or coordination of walking.

So far, motor control and coordination during gait have been studied in postpartum PPP only [40], where large inter-individual differences were reported, and maximum and comfortable walking velocity were found to be reduced. The amplitudes of horizontal pelvis and thorax rotations during gait tended to be larger in postpartum PPP, which is different from low back pain, where these rotations were reported to be normal [13, 15], and clearly opposite to healthy pregnant gait, where rotations tended to be smaller [42]. Furthermore, in postpartum PPP relative phase between horizontal pelvis and thorax rotations was lower at the higher walking velocities, just as in low back pain $[13,15]$, that is to say, pelvis and thorax rotations in the same direction occurred more at the same time.

Recently, the mechanism underlying pelvis-thorax relative phase was investigated in healthy subjects [3]. It was found that pelvis rotations are relatively out-of-phase with the pendular movements of the leg at lower walking velocities, but more in-phase with the leg at higher velocities, while thorax rotations remain more or less outof-phase with the leg at all velocities. This explains why pelvis-thorax relative phase is low at lower walking velocities, and higher at higher velocities. In postpartum PPP this mechanism appears to be altered, but it remains unclear how. Moreover, we do not know if the same phenomenon occurs in PPP during pregnancy.

The aim of the present study was to characterize gait in PPP during pregnancy. First, to see if the findings of the postpartum study could be confirmed for pregnant women with PPP. Second, to see how the coordination of trunk rotations changes in PPP. We compared gait in healthy pregnant women with gait in pregnant women with PPP, focusing on walking velocity, rotational amplitudes, their relative phase, and timing, and inter-subject variability.

\section{Methods}

\section{Selection procedures}

Volunteers were recruited by word of mouth and flyers at the Department of Obstetrics and Gynaecology and the Department of Orthopaedic Surgery (both of the Vrije Universiteit Medical Centre), the Faculty of Human Movement Sciences (Vrije Universiteit), and clinics of Exercise Therapists Mensendieck in Amsterdam. Women who expressed themselves to be interested in the study received an information package. If then they decided to participate, they signed the informed consent statement, and were seen by an orthopaedic surgeon who determined their status as patient or healthy control, and registered age, weight (at the time of the investigation), height, week of pregnancy, parity, and health status. The protocol was approved by the Ethical Committee of the University Hospital.

Included were pregnant women between weeks 20 and 34 , inclusive, and between 20 and 45 years of age, inclusive. Exclusion criteria were orthopaedic or neurological problems with walking other than PPP; surgery of the lumbar spine, pelvis, hip or knee; fracture, malignancy or active inflammation in the lumbar spine or pelvis; ankylosing spondilitis, Scheuermann's kyphosis, active polyarthritis, rheumatoid arthritis, or severe osteoporosis; hormone-induced pregnancy or in vitro fertilization [12]; and/or pulmonary, cardiac, visual, auditive, or cognitive disorders.

The orthopaedic surgeon registered participants as patients if they had pelvic girdle pain as well as problems with daily movements, and if pain was induced by at least one of the following: (1) sacroiliac palpation; (2) manual distraction or (3) compression of the ilia [10]; (4) Active Straight Leg Raising [22]; (5) Posterior Pelvic Pain Provocation [24].

\section{Participants}

Twelve healthy pregnant women and 12 pregnant women with PPP (throughout the paper referred to as "patients") took part in the study. Since heel contact data of one of the patients were missing, we removed all her data, which left us with 11 patients. On unpaired $t$-tests, there were no significant differences between the healthy subjects and the patients, respectively, in age ( 33.1 vs 33.5 years), weight (76.9 vs $74.4 \mathrm{~kg})$, height (1.72 vs $1.68 \mathrm{~m})$, and week (27.0 vs 28.9 ) or number of pregnancy (1.6 vs 1.8 ).

\section{Experimental procedures}

The experimental design and a large part of data processing were similar to those of earlier studies of gait kinematics 
$[3,14,42]$. In order to assess fear of movement, subjects completed the Tampa Scale for Kinesiophobia (TSK) [37]. Patients rated their current pain on a $100 \mathrm{~mm}$ Visual Analogue Scale (VAS).

The experimental task (Fig. 1) consisted of walking on a treadmill (Biostar Giant ${ }^{\mathrm{TM}}$, Biometrics, Almere, The Netherlands) at different velocities. Pelvis, lumbar segment, and thorax rotations were recorded by a $2 \times 3$ camera optoelectronic system $\left(\right.$ OptoTrak $^{\circledR}$, Northern Digital Inc., Waterloo, ON, Canada). Three light metal frames, each with a cluster of three infrared-emitting diodes (Fig. 1, inset), were attached with neoprene bands to the pelvis (between the posterior superior iliac spines), the lumbar spine (at the level of L3), and the thorax (at the level of T6). In front of the subject, an optical flow field, synchronized to the velocity of the belt, was projected on a large screen to mimic walking on a road. To detect heel strike and toe-off, infrared-light emitting markers were placed on the heels and over the fifth metatarsophalangeal joints. The cameras were located $5 \mathrm{~m}$ behind the subject.

To become accustomed to the experimental set-up, subjects walked on the treadmill for $5 \mathrm{~min}$. Then, treadmill velocity was increased with steps of $0.4 \mathrm{~km} / \mathrm{h}$, from 0.6 up to $6.2 \mathrm{~km} / \mathrm{h}$ (a total of 15 "velocity levels"). At each velocity, the participants were asked which velocity was most comfortable, and if the current velocity was too high. If so, the experiment was stopped, and the preceding level designated as their maximum walking velocity. Subjects walked for about $3 \mathrm{~min}$ at each velocity level. When they were accustomed to a new velocity, data were collected for $30 \mathrm{~s}$ at a sampling frequency of $100 \mathrm{~Hz}$.

Data processing: rotational amplitudes

Kinematic data were low pass filtered with a 4th order bi-directional Butterworth filter with a cut-off frequency of $10 \mathrm{~Hz}$. All calculations were carried out with custom-made MatLab (version 6.5) programmes.

We designated positive motion along the $x$-axis as forward, $y$ to the left, and $z$ as upward. For each segment (pelvis, lumbar segment, and thorax), rotations around the $z$-axis ("transverse rotations") were calculated with respect to the global frame of reference. Heel strike was taken to coincide with the point of minimum vertical velocity of the toe marker, and toe-off with its maximum [30]. A stride cycle was defined as the time between two consecutive heel strikes of the same leg.

Pelvis, lumbar segment, and thorax rotational amplitudes were calculated as the absolute angular difference from maximum to minimum rotation within one stride cycle. The differences between these segmental rotations were designated as "spine" rotations, calculated by subtracting the relevant time series from each other: lumbar spine rotation as lumbar segment rotation minus pelvis rotation, thoracic spine rotation as thorax minus lumbar segment, and total spine rotation as thorax minus pelvis. Per velocity level, rotational amplitudes were averaged over all strides.
Fig. 1 Experimental set-up. Subjects walked on a treadmill at predetermined velocities, facing a landscape moving with the same velocity. At the pelvis, lumbar segment, and thorax, cluster markers were attached with neoprene bands. Each cluster marker carried three infrared emitting diodes (inset, bottom right), the movements of which were registered with two sets of three cameras each

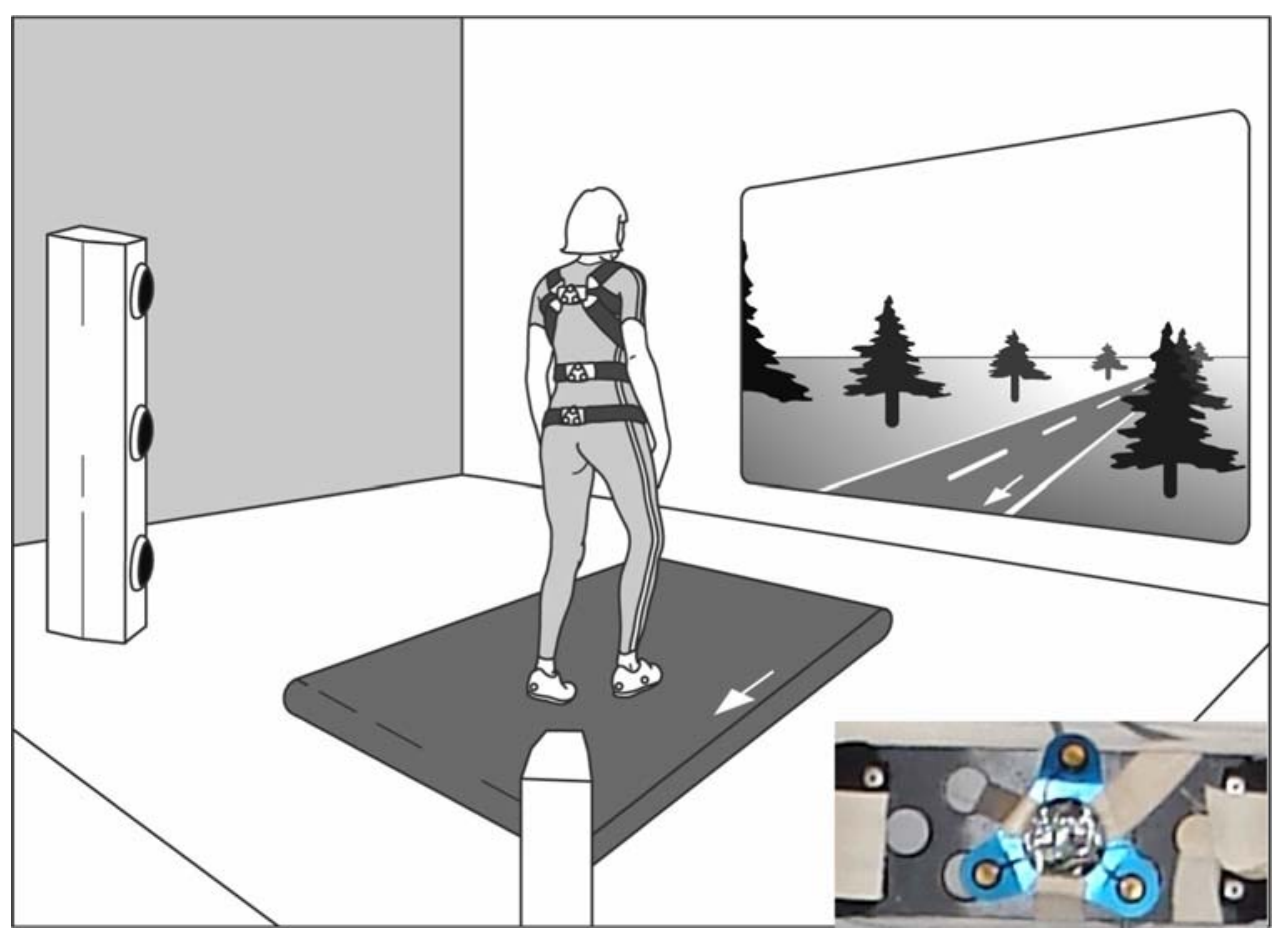


Data processing: timing of rotations

Using a spline interpolation, we time-normalized stride cycles, and calculated an average stride cycle per segment rotation. Then, a sine with period 1 was fitted over it, using a least squares algorithm. In this way, it was ensured that the "timing" of the peak was that of the global pattern, not of a higher harmonic [13]. Thus, we could determine when in the stride cycle the maximum excursion of the segment took place, and if this changed with velocity. Since timenormalization was done with respect to heel contacts, and since angles were defined as described above, values close to 0 would imply that the segment rotated with the pendular movements of the upper leg, values close to 50 that the segment rotated opposite to the upper leg. Note, however, that the legs were not measured directly.

Data processing: relative Fourier phase

From the power spectra of the pelvis, the lumbar segment and the thorax time series, a windowed Fourier Phase was calculated by using a discrete fast Fourier transform algorithm. The window length was four times the period of the first harmonic; it was shifted sample by sample over the entire length of each time series. Pelvis, lumbar segment, and thorax Fourier phases were estimated for each window at the fundamental frequency of the thorax. The signal was then reconstructed in the time domain, yielding a continuous estimate of the Fourier Phase. Continuous Relative Fourier Phase (RFP) was calculated by subtracting the relevant time series: pelvis-lumbar segment RFP as lumbar segment Fourier Phase minus pelvis Fourier Phase, lumbar segment-thorax RFP as thorax minus lumbar segment, and pelvis-thorax RFP as thorax minus pelvis. To calculate mean RFP, circular statistics [7] were used. An RFP of $0^{\circ}$ indicates in-phase coordination, and $180^{\circ}$ anti-phase coordination (where the segments move in opposite directions).

\section{Statistics}

For one-dimensional group comparisons unpaired $t$-tests were used, and for the characterization of pain in the patient group a one sample $t$-test. Pearson correlations were calculated between kinesiophobia (TSK), pain (VAS), comfortable, and maximum walking velocity.

The effect of health status, walking velocity, and their interaction on all velocity-dependent variables was tested with Generalized Estimation Equations (GEE [cf. 16]), which consider measurements within participants as repeated measures. GEE allows for the analysis of designs with missing values-essential since not all patients could walk with all velocities. The interaction term was removed from the model if it proved not to be significant.
To compare inter-individual variability between the groups, we calculated the absolute differences between individual scores and their group means. Over these values, a GEE was conducted. Because in this analysis, we were interested in group differences only, only $P$-values for the effect of group and group $\times$ velocity will be reported, but not for velocity per se.

For $t$-tests, Pearson correlations, and Repeated Measures Analyses of Variance, SPSS (version 14.0) was used, and GEEs were performed with SPIDA (version 6.05). In all statistical procedures, $P<0.05$ was considered significant.

\section{Results}

Fear of movement, pain, and walking velocity

Fear of movement (TSK) was significantly higher in the patients than in the healthy pregnant women (41.8 vs 30.8, $P=0.001$ ). Patients' pain was significantly above 0 (mean VAS-score 44.6, SD 20.7; one sample $t$-test, $P<0.0001)$.

Maximum walking velocity was lower in the patients than in the healthy pregnant women $(4.4 \mathrm{vs} 6.0 \mathrm{~km} / \mathrm{h}, P<$ $0.0001)$. Since none of the patients could walk faster than $5.4 \mathrm{~km} / \mathrm{h}$, we refrained from further analysis at velocities higher than $5.4 \mathrm{~km} / \mathrm{h}$. Comfortable walking velocity was not correlated significantly with age or height [2], and we did not correct for these variables. Mean comfortable walking velocity was lower in the patients $(3.0 \mathrm{vs} 3.7 \mathrm{~km} / \mathrm{h}$, $P=0.005)$.

Within the patient group, the only significant correlation between pain, TSK, and maximum or comfortable walking velocity was found between TSK and maximum walking velocity $\left(r_{\mathrm{P}}=-0.64, P=0.03\right)$.

\section{Rotational amplitudes}

In both groups, pelvis rotational amplitude decreased for velocities up to $3.4 \mathrm{~km} / \mathrm{h}$, to then increase again (Fig. 2a). GEE, revealed a (quadratic) effect of velocity on pelvis rotational amplitude $(P<0.0001)$. The effect of group was also significant $(P<0.0001)$, with larger pelvis rotations in the patients. There was no significant group $\times$ velocity interaction. Inter-individual variability of pelvis rotational amplitudes was significantly larger in the patient group $(P=0.03)$.

In the healthy subjects, lumbar segment rotational amplitude (Fig. 2b) decreased for velocities up to $4.4 \mathrm{~km} / \mathrm{h}$, to then remain more or less stable; in the patients, the pattern was more irregular. Overall, the effect of velocity was significant $(P<0.0001)$, as was the effect of group $(P=0.001)$, patients having larger lumbar rotations. There was no significant group $\times$ velocity interaction in lumbar 

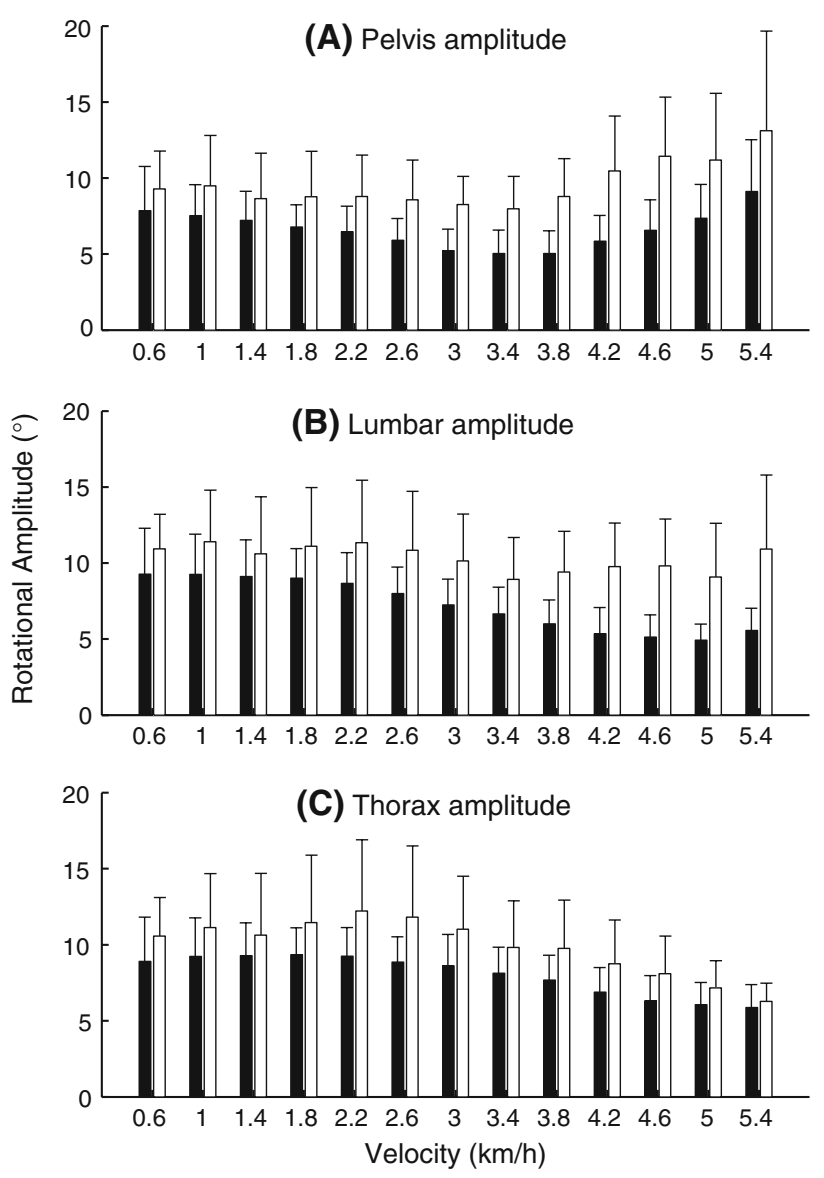

Fig. 2 Pelvis (a), lumbar segment (b), and thorax (c) transverse rotational amplitudes in the healthy pregnant women (black) and the pregnant women with PPP (white). Error bars represent standard deviations

segment rotational amplitude per se, but there was such an interaction $(P=0.04)$ in the inter-individual variability of lumbar segment rotational amplitude, with larger variability in the patient group at the higher velocities.

Thorax rotational amplitude (Fig. 2c) decreased from $2.0 \mathrm{~km} / \mathrm{h}$ onwards for both groups; this effect was significant $(P<0.0001)$. Moreover, thorax rotational amplitude showed an effect of group $(P=0.01)$, again patients having larger rotations. No significant group $\times$ velocity interaction was found. Inter-individual variability of thorax rotational amplitude was significantly larger in the patient group $(P=0.04)$.

Rotations between the above segments (Fig. 3), i.e., between the pelvis and the lumber segment (lumbar spine rotation), between the lumbar segment and the thorax (thoracic spine rotation), and between the pelvis and the thorax (total spine rotation), increased significantly with increasing velocity $(P$-values $<0.0001)$, but there was no significant effect of group, nor a significant group $\times$

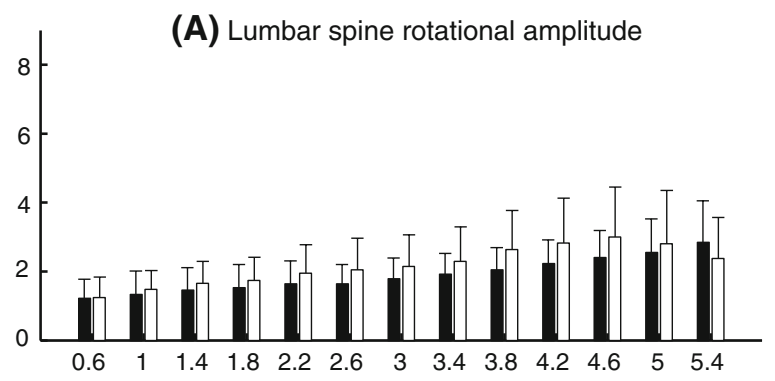

(B) Thoracic spine rotational amplitude
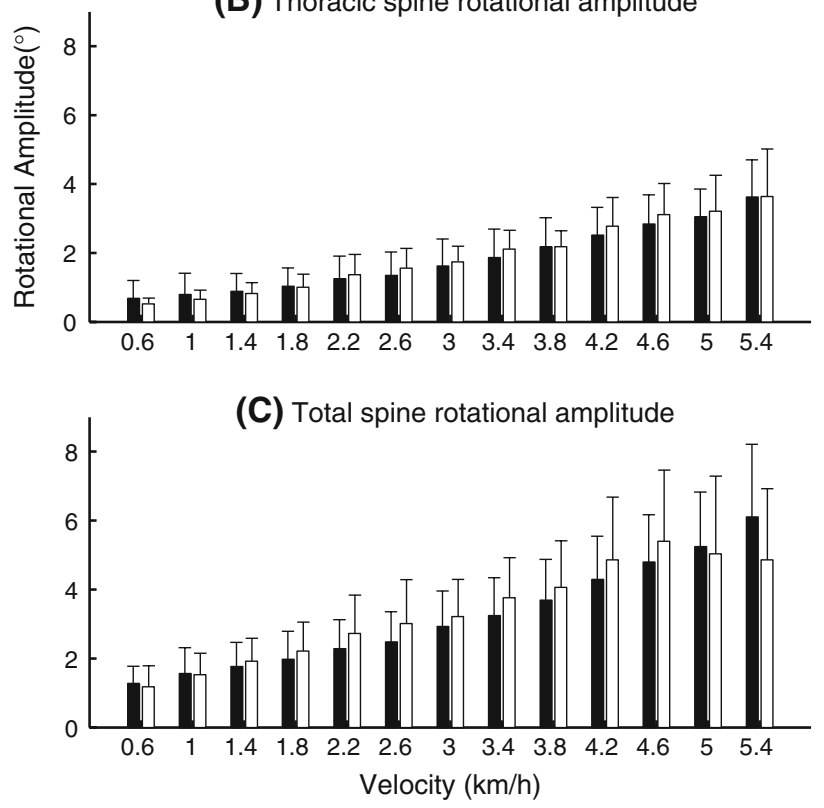

Fig. 3 Lumbar spine (a), thoracic spine (b), and total spine (c) transverse rotational amplitudes in the healthy pregnant women (black) and the pregnant women with PPP (white). Error bars represent standard deviations

velocity interaction. The inter-individual variability of these spinal rotations did not differ between groups.

Timing of rotations

The timing of pelvis rotations (Fig. 4a) changed significantly with increasing velocity $(P<0.0001)$. From about $2.2 \mathrm{~km} / \mathrm{h}$ onwards the peak of pelvis rotation shifted towards the beginning of the stride cycle. There was no significant effect of group or group $\times$ velocity interaction in the timing of pelvis rotations.

The timing of lumbar rotations (Fig. 4b) showed almost the same pattern, but lumbar rotations started to change somewhat later, from about $3.0 \mathrm{~km} / \mathrm{h}$ onwards. The effect of velocity on the timing of lumbar rotations was significant $(P<0.0001)$. No significant effects of group or group $\times$ velocity interaction were found.

Overall, the timing of thorax rotations (Fig. 4c) showed no significant effect of velocity or group, but there was a 


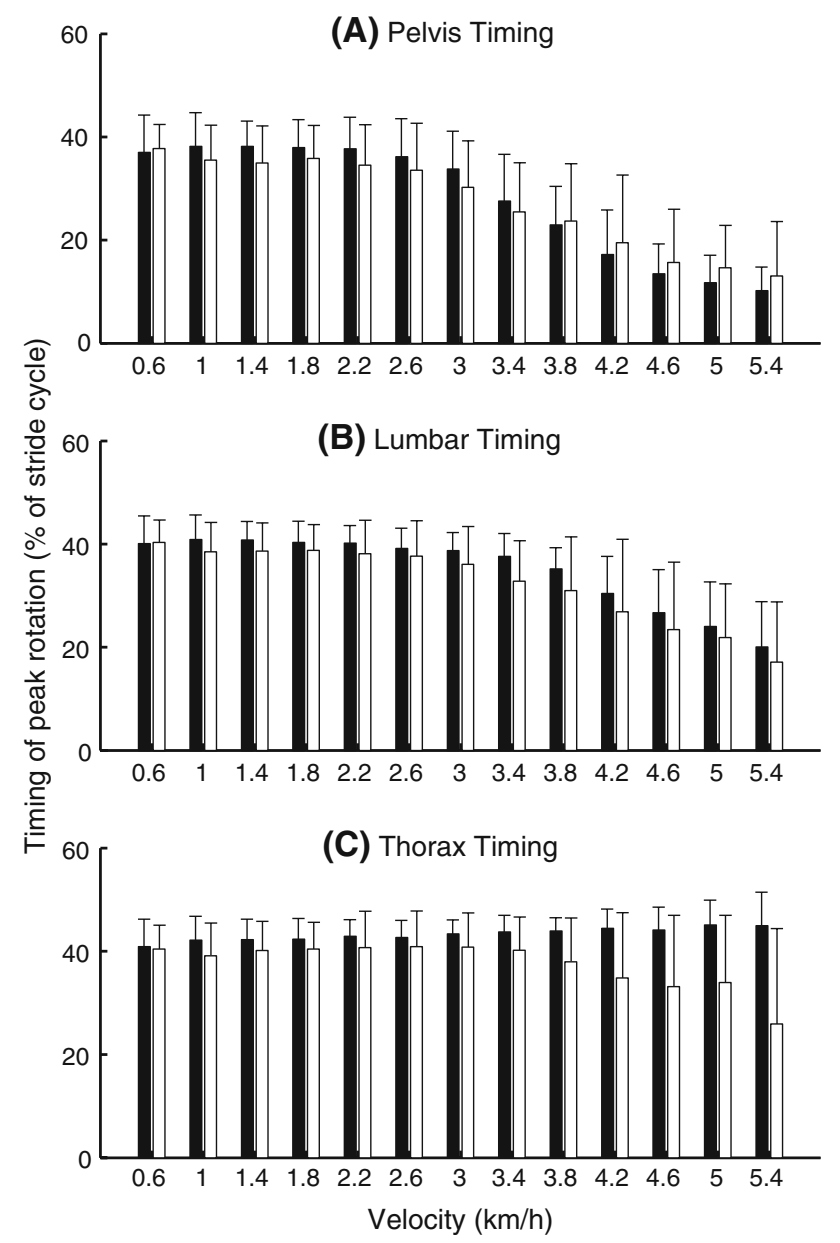

Fig. 4 Timing of pelvis (a), lumbar segment (b), and thorax (c) peak transverse rotations. Values closer to 0 imply that the rotation was more in synchrony with the upper leg, while values closer to 50 imply that the rotation was more in opposition to the movements of the upper leg. Black bars represent data from the healthy pregnant women, white from the pregnant women with PPP. Error bars represent standard deviations

significant group $\times$ velocity interaction $(P=0.04)$. With increasing velocity, the patient group shifted the peak of their thorax rotation more towards the beginning of the stride cycle, just as the pelvis and the lumbar segment.

The inter-individual variability of the timing of the pelvis and the lumbar segment revealed no significant effects of group or group $\times$ velocity interaction, but there was a significant group $\times$ velocity interaction in the variability of the timing of thorax rotations $(P=0.02)$, the patient group having larger variability at the higher velocities.

\section{Relative Fourier phase}

Relative phase between the pelvis and the lumbar segment increased for velocities up to $4.2 \mathrm{~km} / \mathrm{h}$, to then decrease
(Fig. 5a). The effect of velocity on pelvis-lumbar relative phase was significant $(P<0.0001)$. There was no significant effect of group, but a significant group $\times$ velocity interaction was present $(P=0.008)$, patients having a lower relative phase at the higher velocities.

Lumbar segment-thorax relative phase (Fig. 5b) increased with increasing velocity $(P<0.0001)$. There was also a significant effect of group $(P<0.0001)$, with lower relative phase in the patients, and a significant group $x$ velocity interaction $(P<0.0001)$, patients' relative phase remaining lower at the higher velocities.

A very similar pattern, with somewhat higher values, was found for pelvis-thorax relative phase (Fig. 5c), with significant effects of velocity, group, and group $\times$ velocity $(P$-values $<0.0001)$.

There were no group effects or group $\times$ velocity interactions in the inter-subject variability of any of the relative phase measures.
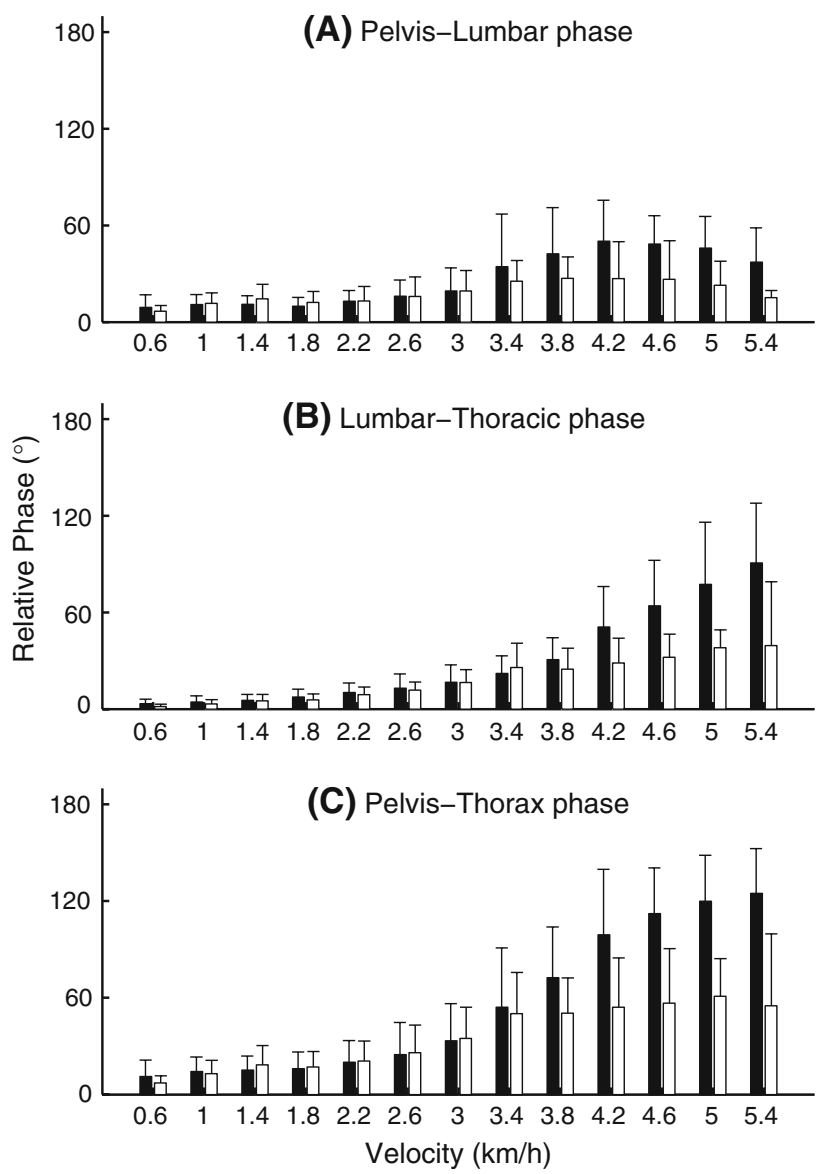

Fig. 5 Pelvis-lumbar segment (a), lumbar segment-thorax (b), and pelvis-thorax (c). Relative Fourier Phase in the healthy pregnant women (black) and the pregnant women with PPP (white). Error bars represent standard deviations 


\section{Discussion}

We compared healthy pregnant women with pregnant women who were suffering from Pregnancy-related Pelvic girdle Pain (PPP). In our study, the women with PPP were more afraid of movement than healthy women, were in pain, and had lower comfortable as well as maximum walking velocity. Among these variables, there was a significant negative correlation between fear and maximum walking velocity.

To the best of our knowledge, this is the first time that fear of movement was assessed in PPP. Walking velocity, on the other hand, was previously investigated, and reported to be lower [e.g., 41-42]. Walking slowly appears to be a general characteristic of locomotor pathologies, while its exact causes remain largely unknown. Of course, an inability to generate the moments and forces necessary for walking may result in slow walking, but other than biomechanical factors may also play a role. Our current results suggest that fear, rather than pain itself, may be a factor that limits maximum walking velocity in PPP [cf. 18].

\section{Segmental rotational amplitudes}

We found increased rotational amplitudes of the pelvis, the lumbar segment, and the thorax in the patient group. For the pelvis and the thorax similar findings, albeit not significant, were reported earlier for postpartum PPP [40]. Still, this result may seem surprising since the patients suffer from pain in their pelvic girdle so that one could expect them to move their pelvis less. Nor is it likely that fear of movement would lead to an increase in transverse pelvic rotations during gait.

There was more inter-subject variability in all three rotational amplitudes in the patients, which appears to suggest that different patients select different strategies. Post hoc we inspected the relevant graphs and found in the patients that extreme values in one amplitude tended to coincide with extreme values in the other amplitudes, which did not appear to be the case in the healthy controls. Patients sometimes told us that they changed their walking strategy by consciously rotating the trunk, which may be what we see here. We know that PPP patients have problems with hip flexion during walking [35], a circumstance possibly inviting patients to try-out different walking strategies. An alternative explanation would be that hip endorotator weakness [23] leads to more exorotation and thereby larger amplitudes of pelvis rotations [3], but then it would need to be explained why the lumbar segment and the thorax follow suit.

Rotational amplitudes of the spine

Notwithstanding the fact that rotations of the pelvis, the lumbar segment, and the thorax were larger in the patients, rotations between these segments failed to show any effect of group, that is, we found no indications of a group effect on the rotational amplitudes of the lumbar spine, the thoracic spine, or the total spine. Spinal rotations did increase with velocity, but in a similar way for both groups. Note that the rotational amplitudes we found remained well below the passive range of trunk motion as reported in the literature [1, 19].

The timing of rotations

In both groups, we found that the time of maximum pelvis rotation moved more towards the beginning of the cycle at higher velocities, as is normal in healthy non-pregnant subjects [3]. Although not studied earlier, it may be expected that rotations of the lumbar segment follow this pattern, as we found in the present study for both groups. In normal healthy subjects, the time of maximum thorax rotation remains unchanged, halfway the cycle, as in the controls of the present study. In our patient group, however, maximum thorax rotation occurred earlier at the higher velocities, "attracted", one could say, by the rotations of the pelvis and the lumbar segment. A similar strategy was also found in low back pain [e.g., 15, Fig. 1, left panel]. In the present study, inter-subject variability of the timing of thorax rotations was larger in the patient group at higher velocities, indicative, again, of differences between individual adaptative strategies.

\section{Relative Fourier phase}

Relative phase between the pelvis and the lumbar segment had an inverted U-shape, apparently because the lumbar segment starts to change the timing of its peak rotation at somewhat higher velocities than the pelvis (Fig. 5). This was more or less the same in both groups. Between the pelvis and the thorax, as well as between the lumbar segment and the thorax, relative phase was larger in the healthy controls, especially at the higher velocities. For pelvis-thorax relative phase, this has been reported earlier for post-partum PPP [40].

\section{An interpretation}

The four major results of our present study are (A) segmental rotations were larger in patients, (B) spinal rotations were not larger in patients, (C) thorax timing moved towards earlier in the stride cycle at higher velocities in patients, (D) pelvis-thorax and lumbar segment-thorax relative phase were lower at the higher velocities in patients.

Since phase-relationships were non-zero, result A would lead to the opposite of B, unless something else changed, 
that is, C and/or D. We propose the following interpretation. Some patients select gait strategies with larger rotations (A) to overcome their problems with walking, and then avoid larger spinal rotations (B) by adjusting the timing of the thorax at higher velocities (C), leading to a reduction of relative phase (D). If this interpretation is correct, it would remain to be investigated why strategies with larger rotations appear to be so attractive, if such strategies do in fact deal with the underlying problems, and what the major disadvantages of such strategies would be, if any.

Note that we found more variability in the patient group for rotational amplitudes (A) and the timing of thorax rotations $(\mathrm{C})$, but not in the other variables. We believe that if the nervous system is confronted with a problem, adaptive strategies are stochastically produced and then selected-"contingent adaptation" [36]. Thus, in our interpretation, the fact that more variability was seen in rotational amplitudes and the timing of thorax rotations, can be taken as a sign that these are the variables used by the nervous system to adapt to the problems at hand.

In low back pain, larger segmental rotations have not been reported so far [13, 14], while pelvis-thorax coordination appear to be altered in the same way as in the present study, and a published Figure [15] suggests that the timing of thorax peak rotation changes in the same direction as we found for the PPP patients. If confirmed, this would have two important implications. First, that walking with larger pelvis rotations (followed by similar lumbar segment and thorax rotations) is specific to PPP. Second, that the adaptation in the timing of the thorax, hence in relative phase, is not specific to either PPP or low back pain, but rather a general preventive mechanism to avoid excessive torsional strain in the spine and/or the sacroiliac joints.

\section{Limitations of the present study}

In order to control velocity, we used treadmill walking, which may have an overall [39], but most likely no differential effect on gait kinematics. Sample sizes in the present study were small, but still, we found significant differences, suggesting these differences to be large.

\section{Clinical implications}

A variety of changes in motor control and coordination have been reported for PPP or pregnancy-related low back pain [e.g., 4, 33-34, 42]. In the present study, gait was found to be altered, not only is it slower, but at least some of the patients walk with larger pelvis, lumbar segment, and thorax rotations, possibly as an adaptation to underlying problems. The risk of increasing torsion in the sacro- iliac joints or the spine, appears to be under control by changing the timing of peak thorax rotations so that they occur earlier in the stride cycle at higher velocities, leading to lower pelvis-thorax and lumbar segment-thorax relative phase at higher velocities.

It has been suggested that such a walking pattern is less stable than normal walking [3], which could lead to higher energy consumption, not analysed in the present study, and a feeling of being insecure, as corroborated in the present study by the fact that the patients, in general, were afraid of movement, which may be [18] related to the limitation of maximum walking velocity in PPP.

The fact that some still regard PPP as a "fashionable disease" [31] may contribute to the discomfort of the women in question, and any objective finding, such as in the present study, will help to taking patients seriously. Exercise therapy and acupuncture were reported effective in PPP [29], but the effects are small, and there is a lack of high-quality studies. We propose to use changes in walking kinematics as dependent variables in higher quality intervention studies. Last but not least, exercise therapists should know that women with PPP may avoid excessive spinal rotation during gait. As long as such avoidance is adaptive, exercise should exploit it. On the other hand, if the altered gait pattern persists after the original trauma has healed, patients have to relearn normal walking.

\section{Conclusion}

The results of the earlier study on postpartum PPP were confirmed for PPP during pregnancy: lower walking velocity, larger horizontal rotations during gait, and a reduced relative phase between these rotations at the higher walking velocities. Moreover, the mechanism underlying this lagging behind of relative phase is becoming clear: an earlier peak of thorax rotations at higher velocities appears to reduce relative phase in PPP, probably to avoid excessive rotational torque in the sacroiliac joints and the spine.

Acknowledgements This paper is dedicated to the memory of Paul I.J.M. Wuisman. The authors gratefully acknowledge financial support from the Dutch Society of Exercise Therapists Mensendieck, the Mensendieck Development Foundation, Stryker/Howmedica, Program for New Century Excellent Talents in Fujian Province University, and Biomet Nederland. The experiment complied with the current laws of The Netherlands, inclusive of ethics approval. Gert Kwakkel, Robin Zwart, Roel Divendal, Rolf van de Langenberg, Sander de Wolf, and Juan Shang assisted with the measurements. Mianlai Zhou and Yinwei Zhan were helpful in setting up the data analysis system. Jaap Harlaar (Department of Rehabilitation, VU Medical Centre), Hanneke de Vries (Department of Obstetrics and Gynaecology), and Kimi Uegaki (Faculty of Human Movement Sciences, Vrije Universiteit) contributed to the writing of an early version of the manuscript, Mark Scheper to the writing of the last 
version. We appreciated discussions with Hans Christian Östgaard, Jan Mens, Robert Dantzer, Paul Hodges, and Hans van den Berg.

Open Access This article is distributed under the terms of the Creative Commons Attribution Noncommercial License which permits any noncommercial use, distribution, and reproduction in any medium, provided the original author(s) and source are credited.

\section{References}

1. Boden A, Öberg K (1998) Torque resistance of the passive tissues of the trunk at axial rotation. Appl Ergon 29:111-118. doi: 10.1016/S0003-6870(97)00030-6

2. Bohannon RW (1997) Comfortable and maximum walking speed of adults aged 20-79 years: reference values and determinants. Age Ageing 26:15-19. doi:10.1093/ageing/26.1.15

3. Bruijn SM, Meijer OG, Van Dieën JH, Kingma I, Lamoth CJC (2008) Coordination of leg swing, thorax rotations, and pelvis rotations during gait: the organisation of total body angular momentum. Gait Posture 27:455-462. doi:10.1016/j.gaitpost. 2007.05.017

4. Commissaris DA, Nilsson-Wikmar LB, Van Dieen JH, Hirschfeld $\mathrm{H}$ (2002) Joint coordination during whole-body lifting in women with low back pain after pregnancy. Arch Phys Med Rehabil 83:1279-1289. doi:10.1053/apmr.2002.33641

5. Damen L, Buyruk HM, Guler-Uysal F, Lotgering FK, Snijders CJ, Stam HJ (2001) Pelvic pain during pain is associated with asymmetric laxity of the sacroiliac joints. Acta Obstet Gynecol Scand 80:1019-1024. doi:10.1034/j.1600-0412.2001.801109.x

6. Fast A, Shapiro D, Ducommun EJ, Friedmann LW, Bouklas T, Floman Y (1987) Low-back pain in pregnancy. Spine 12:368371. doi:10.1097/00007632-198705000-00011

7. Fisher NI (1993) Statistical analysis of circular data. Cambridge University Press, Cambridge

8. Hansen A, Jensen DV, Wormslev M, Minck H, Johansen S, Larsen EC, Wilken-Jensen C, Davidsen M, Hansen TM (1999) Symptom-giving pelvic girdle relaxation in pregnancy, II: symptoms and clinical signs. Acta Obstet Gynecol Scand 78:111115. doi:10.1034/j.1600-0412.1999.780207.x

9. Kristiansson P, Svärdsudd K (1996) Discriminatory power of tests applied in back pain during pregnancy. Spine 21:2337-2343. doi:10.1097/00007632-199610150-00006

10. Kristiansson P, Svärdsudd K, Von Schoultz B (1996) Back pain during pregnancy: a prospective study. Spine 21:702-709. doi: 10.1097/00007632-199603150-00008

11. Kristiansson P, Svärdsudd K, Von Schoultz B (1996) Serum relaxin, symphyseal pain, and back pain during pregnancy. Am J Obstet Gynecol 175:1342-1347. doi:10.1016/S0002-9378(96) 70052-2

12. Kristiansson P, Nilsson-Wikmar L, Von Schoultz B, Svärdsudd K, Wramsby H (1998) Back pain in in-vitro fertilized and spontaneous pregnancies. Hum Reprod 13:3233-3238. doi: 10.1093/humrep/13.11.3233

13. Lamoth CJC, Meijer OG, Wuisman PIJM, Van Dieën JH, Levin MF, Beek PJ (2002) Pelvis-thorax coordination in the transverse plane during walking in persons with nonspecific low back pain. Spine 27:E92-E99. doi:10.1097/00007632-200202150-00016

14. Lamoth CJC, Daffertshofer A, Meijer OG, Moseley GL, Wuisman PIJM, Beek PJ (2004) Effects of experimentally induced pain and fear of pain on trunk coordination and back muscle activity during walking. Clin Biomech 19:551-563. doi: 10.1016/j.clinbiomech.2003.10.006
15. Lamoth CJC, Daffertshofer A, Meijer OG, Beek PJ (2006) How do persons with chronic low back pain speed up and slow down? Trunk-pelvis coordination and lumbar erector spinae activity during gait. Gait Posture 23:230-239. doi:10.1016/j.gaitpost. 2005.02.006

16. Liang KY, Zeger SL (1993) Regression analysis for correlated data. Annu Rev Public Health 14:43-68. doi:10.1146/annurev.pu. 14.050193.000355

17. MacLennan AH, MacLennan SC, The Norwegian Association for Women with Pelvic Girdle Relaxation (1997) Symptom-giving pelvic girdle relaxation of pregnancy, postnatal pelvic joint syndrome and developmental dysplasia of the hip. Acta Obstet Gynecol Scand 76:760-764. doi:10.3109/00016349709024343

18. Maki BE (1997) Gait changes in older adults: predictors of falls or indicators of fear. J Am Geriatr Soc 45:313-320

19. McGill S, Seguin J, Bennett G (1994) Passive stiffness of the lumbar torso in flexion, extension, lateral bending, and axial rotations: effect of belt wearing and breath holding. Spine 19:696-704

20. Meijer OG, Van Dieën JH, Hu H, Bruijn S, Ten Cate A, Leijer J-P, Wuisman P, Mallant M, Comans E, Nanayakkara P (2006) Contingente adaptatie: De rationale voor oefentherapie [Contingent adaptation: the rationale for exercise therapy]. Beweegreden September:20-29

21. Mens JMA, Vleeming A, Stoeckart R, Stam HJ, Snijders CJ (1996) Understanding peripartum pelvic pain: implications of a patient survey. Spine 21:1363-1369. doi:10.1097/00007632199606010-00017

22. Mens JMA, Vleeming A, Snijders CJ, Stam HJ, Ginai AZ (1999) The active straight leg raising test and mobility of the pelvic joints. Eur Spine J 8:468-474. doi:10.1007/s005860050206

23. Mens JMA, Vleeming A, Snijders CJ, Ronchetti I, Stam HJ (2002) Reliability and validity of hip adduction strength to measure disease severity in posterior pelvic pain since pregnancy. Spine 27:1674-1679. doi:10.1097/00007632-200208010-00017

24. Östgaard HC, Zetherström G, Roos-Hansson E (1994) The posterior pelvic pain provocation test in pregnant women. Eur Spine J 3:258-260. doi:10.1007/BF02226575

25. Östgaard HC, Zetherström G, Roos-Hansson E, Svanberg B (1994) Reduction of back and posterior pelvic pain in pregnancy. Spine 19:894-900

26. O'Sullivan PB, Beales DJ (2007) Diagnosis and classification of pelvic girdle pain disorders, Part I: a mechanism based approach within a biopsychosocial framework. Man Ther 12:86-97. doi: 10.1016/j.math.2007.02.001

27. O'Sullivan PB, Beales DJ (2007) Diagnosis and classification of pelvic girdle pain disorders, Part II: illustration of the utility of a classification system via case studies. Man Ther 12:E1-E12. doi: 10.1016/j.math.2007.03.003

28. Padua L, Padua R, Bondi R, Ceccarelli E, Calandro P, D'Amico P, Mazza O, Tonali P (2002) Patient-oriented assessment of back pain in pregnancy. Eur Spine J 11:272-275. doi:10.1007/ s005860100262

29. Penninck VE, Young G (2007). Interventions for preventing and treating pelvic and back pain in pregnancy. Cochrane Database Syst Rev CD 001139

30. Pijnappels M, Bobbert MF, Van Dieën JH (2001) Changes in walking pattern caused by the possibility of a tripping reaction. Gait Posture 14:11-18. doi:10.1016/S0966-6362(01)00110-2

31. Renckens C (2004) Dwaalwegen in de geneeskunde [Medicine led astray]. Bert Bakker, Amsterdam

32. Röst CCM, Jacqueline J, Kaiser A, Verhagen AP, Koes BW (2004) Pelvic pain during pregnancy: a descriptive study of signs and symptoms of 870 patients in primary care. Spine 29:25672572. doi:10.1097/01.brs.0000145416.22782.9 
33. Sihvonen T, Huttunen M, Makkonen M, Airaksinen O (1998) Functional changes in back muscle activity correlate with pain intensity and prediction of low back pain during pregnancy. Arch Phys Med Rehabil 79:1210-1212. doi:10.1016/S0003-9993(98) 90264-7

34. Stuge B, Mørkved S, Haug Dahl H, Völlestad N (2006) Abdominal and pelvic floor muscle function in women with and without long lasting pelvic girdle pain. Man Ther 11:287-296. doi:10.1016/j.math.2005.07.003

35. Sturesson B, Udén G, Udén A (1997) Pain pattern in pregnancy and "catching" of the leg in pregnant women with posterior pelvic pain. Spine 22:1880-1883. doi:10.1097/00007632199708150-00013

36. Van Dieën JH (2007) Low-back pain and motor behavior: contingent adaptations, a common goal. In: Sixth interdiscipinary world congress on low back \& pelvic pain: diagnosis and treatment, the balance between research and clinic, Barcelona, November 7-10, pp 3-14

37. Vlaeyen JW, Kole-Snijders AM, Boeren RG, Van Eek H (1995) Fear of movement/(re)injury in chronic low back pain and its relation to behavioral performance. Pain 62:363-372. doi: 10.1016/0304-3959(94)00279-N

38. Vleeming A, De Vries HJ, Mens JMA, Van Wingerden JP (2002) Possible role of the long dorsal sacroiliac ligament in women with peripartum pelvic pain. Acta Obstet Gynecol Scand 81:430 436. doi:10.1034/j.1600-0412.2002.810510.x

39. Vogt L, Pfeifer K, Banzer W (2002) Comparison of angular lumbar spine and pelvis kinematics during treadmill and overground locomotion. Clin Biomech 17:162-165. doi:10.1016/ S0268-0033(01)00111-5

40. Wu W, Meijer OG, Jutte PC, Uegaki K, Lamoth CJC, De Wolf GS, Van Dieën JH, Wuisman PIJM, Kwakkel G, De Vries JIP, Beek PJ (2002) Gait in patients with pregnancy-related pain in the pelvis: an emphasis on the coordination of transverse pelvic and thoracic rotations. Clin Biomech 17:678-686. doi: 10.1016/S0268-0033(02)00109-2

41. Wu WH, Meijer OG, Uegaki K, Mens JMA, Van Dieën JH, Wuisman PIJM, Östgaard HC (2004) Pregnancy-related pain in the pelvis (PPP), I: terminology, clinical presentation, and prevalence. Eur Spine J 13:575-589. doi:10.1007/s00586-003-0615-y

42. Wu W, Meijer OG, Lamoth CJC, Uegaki K, Van Dieën JH, Wuisman PIJM, De Vries JIP, Beek PJ (2004) Gait coordination in pregnancy: transverse pelvic and thoracic rotations and their relative phase. Clin Biomech 19:480-488. doi:10.1016/ j.clinbiomech.2004.02.003 